\title{
EASR: Graph-based Framework for Energy Efficient Smart Routing in MANET using Availability Zones
}

\author{
Ramanna Havinal*, Girish V. Attimarad**, M. N. Giri Prasad*** \\ * Department of Electronics \& Communication Engineering. College of Engineering, Ambajogai, India \\ ** Department of Electronics \& Communication Engineering, Dayanand Sagar College of Engg, Bengaluru, India \\ *** Department of Electronics \& Communication Engineering, JNTUA College of Engg. Anantapuramu, India
}

\begin{tabular}{l} 
Article Info \\
\hline Article history: \\
Received Jul 4, 2015 \\
Revised Sep 11, 2015 \\
Accepted Sep 29, 2015 \\
\hline
\end{tabular}

\section{Keyword:}

AODV

Availability zones

Energy issues

Mobile adhoc network

Routing protocol

\begin{abstract}
Energy consumption in Mobile Adhoc Network (MANET) is a topic of research from more than a decade. Although there are multiple archival of literatures, that have proposed various energy-efficient algorithms for reducing the energy consumption to improve energy efficiency. Establishing correct and reliable route is an important design issue in MANET, but a more challenging goal is to provide an energy efficient route. But, it was observed that the majority of such energy efficient routing protocols just gives a symptomatic solution which addresses and mitigated the energy issues overlooking various associated issues like quality of services. Moreover, in the majority of research previous studies, it is found that AODV and DSDV are highly in adoption rate among the researcher for solving energy issues using routing protocols. This manuscript after reviewing some of the significant literatures in past explored issues in existing AODV and DSDV and proposes a novel energy efficient routing protocol by incorporating a new actor called an availability zone. The proposed model shows better energy efficiency and QoS compared to AODV and DSDV.
\end{abstract}

Copyright (c) 2015 Institute of Advanced Engineering and Science. All rights reserved.

\section{Corresponding Author:}

Ramanna Havinal,

Department of Electronics and Communication Engineering,

M B E Society's College of Engineering, Ambajogai, Maharashtra, India

Email:rshavinal@gmail.com

\section{INTRODUCTION}

A Mobile Adhoc Network (MANET) is an infrastructure less networking system that comprises of a large number of mobile nodes which are interconnected with each other using adhoc networking mechanism [1]. Each mobile node is itself considered as routers. Owing to the inherent features of dynamic topology, every node in MANET depletes its energy while attempting to establish a communication. In the past various routing protocols have been introduced that targets to optimize the energy required for performing communication among the mobile nodes. One of the interesting observations about the energy efficiency in literature archival for MANET is that the majority of the energy aware techniques were basically evaluated on wireless sensor network where optimal energy is a big challenge for enhancement [2]. However, such techniques cannot be thought of implementing on energy issues in MANET, as a problem space for MANET highly differs from a wireless sensor network. In MANET, the mobile node has better resource availability as compared to wireless sensor network. Therefore, in order to mitigate the energy issues in MANET, various associated sub-problems should also be simultaneously addressed and routing protocol is one of it. An energy aware routing protocol must ensure better quality of service, effective security, and extensive scalability. The essential goal of energy efficient routing protocols in MANET is basically to enhance the overall communication performance and to retain extensive network lifetime as well.A number of protocols have been proposed to reduce the energy consumption 
It has been seen that existing routing protocols that ensures the energy efficiency attempts to minimize the energy.It has been seen that existing routing protocols that ensures the energy efficiency attempts to minimize the energy dissipation using various attributes of routing and thereby ensure energy effectiveness. If the aspects of energy awareness or energy efficiency are considered in the design of a routing protocol as done in many literatures, it can be said that the presented solution is only symptomatic. By the term 'symptomatic', it means that the solution addresses only energy issues and doesn't cover up other associated issues that arise due to energy depletion and dynamic topology in MANET.

Hence, the proportion of effectiveness in solution is always less in energy effective routing protocols in MANET. Another reason may be the assumptions and consideration of the routing strategy being discussed. We believe that if MANET routing protocols are researched from more than a decade with almost similar implementation of AODV, DSDV, DSR, CSR [3] as the most preferred choice for route design. This perception should be changed and researchers should be motivated for coming up with an entirely new routing concept. Majority of the literatures are found with only enhancement to the existing routing protocols for which reason, the problems are not completely mitigated. Hence, this paper is a deliberate attempt to introduce a highly novel routing framework using the advanced graph theory for the purpose of energy efficiency. The model discusses about an actor called as availability zone, keeping in viewpoint of futuristic application of MANET for the purpose of robust and reliable routing scheme that ensures energy efficiency. The discussion of Introduction in Section 1 is followed by Section 1.1 that highlights about background of study followed by problem discussion in Section 1.2. Section 1.3 introduces the proposed model followed by elaborated discussion of research methodology in Section 2. Section 3 discusses about the Result and discussion being accomplished from the study along with comparative performance analysis that is further followed by conclusion in Section 4 .

\subsection{Background}

This section discusses about all the prominent research work carried out to mitigate energy problems in MANET most recently. Shivashankar et al [4] proposed efficient Power Aware Routing (EPAR), a new power aware routing protocol that increases the network lifetime of the MANET. The author has also evaluated three ad hoc network routing protocols (EPAR, Minimum Transmission Power Routing and Dynamic Source Routing) in different network scales taking into consideration the power consumption. The outcome of the study was found to reduce for more than $20 \%$ the total energy consumption and decreases the mean delay, especially for high load networks while achieving a good packet delivery ratio. Aydogdu and Karasan [5] proposed an analytical model for the IEEE 802.11 Distributed Coordinated Function in multihop adhoc networks that considers hidden terminals and accurately works for an outsized vary of traffic load that are used to investigate the energy consumption of varied relaying methods. They gave truth of the present analytical frameworks for IEEE 802.11 systems were insufficient for an energy conservation analysis in wireless multi-hop networks. They took major attributes of the proposed Distributed Coordinated Function (DCF) model beside the constraints and assumptions of the previous DCF models. The energy-efficiency over a large varies of situations. The given results show that the energy economical routing strategy depends not solely on the processing power, however conjointly depends on the traffic load.

Abdulla et al. in [6] have presented a unique routing technique for ensuring energy aware routing policies in adhoc network. The authors have investigated the issue of choosing the optimal transmission distance to minimize the energy consumption of wireless ad hoc networks. Seung Hwan Lee et.al in [7], proposed energy efficient power management mechanism for base station in mobile communication systems and an economical sector power management based mostly on the distance between the base station and the mobile node. They additionally proposed a sleep mode energy management mechanism, where every sector monitors the amount of user in sector cell. if the variety of mobile node falls down a given threshold in sector cell, base station shuts down power.

Zuo et al. [8] proposed a cross-layer operation aided energy efficient opportunistic routing algorithm for Ad Hoc networks and an energy consumption-based objective function combined with power allocation, which is employed both for finding a theoretical bound and for conveying the packets through the network. Ali et al. [9] have developed an energy oriented power aware approach for optimizing the energy consumption per node so as not to exhaust some or any of the nodes/ network path. Mukherjee et al. [10] proposes a self-managing, energy-efficient multicast routing suite based on the self-stabilization paradigm. The framework considers the link state change rate, application data traffic intensity, application packet delivery requirements, and the stabilization latency. Mangai and Tamilrasi [11] have presented an Improved Location aided Cluster based Routing Protocol for GPS enabled MANETs using cluster and position information. The authors have also presented an algorithm for inter-cluster as well as intra-cluster routing. The simulation study is carried out in NS2 using MAC layer protocol. The outcome of the study was evaluated with respect to end-to-end delay, packet delivery ratio, and control overhead. The paper has 
presented a unique routing protocol that becomes functional by exchanging positional information. The outcome of the study found a 30\% reduction in control overhead compared to the existing techniques. Correia and Vazao [12] proposed a routing algorithm based on simple ant colony optimization technique. The presented technique consists of route discovery, maintenance, selection, and route repair. The study uses the broadcasting mechanism of the neighbor nodes. However, the technique suffers from issues of increased time in the route discovery process. The only advantage of the technique is that it to reduce $25 \%$ of the routing overhead as compared to AODV.

Fu et al. [13] propose a local capacity constrained density adaptive routing algorithm for large scale vehicular DTN in urban areas which targets to increase the packet delivery ratio within deadline. The core idea is to adjust forward strategy and protocol parameters based on the local node density with the awareness of local channel capacity. A utility value, suggesting the delivery probability of the packets within time constraint is maintained for each packet by each mobile node. Li et al. [14] carried out a theoretical analysis of traffic load distribution in circular sailing routing mechanism in the multi hop wireless network. This study is similar to routing protocols of MANET by evaluating the traffic load at any point in the uniform traffic. The study essentially focuses on the a concept called as circular sailing routing maps nodes on the surface of a sphere and select routes based on surface distances. Jianli et al. [15] propose a scheme called HEAD (a hybrid mechanism to enforce node cooperation in mobile ad hoc networks) to make the misbehavior unattractive. HEAD is an improvement to OCEAN (observation-based cooperation, enforcement in adhoc networks). It employs only first hand information and works on the top of DSR (dynamic source routing) protocol. Roy et al. [16] proposes routing strategy can be optimized to establish multiple stable paths between source and destination nodes satisfying user's QoS requirement which need to be maintained consistency

Liu et al. [17] propose Receiver Consensus, which exploits geographical information to help nodes autonomously achieve agreement on forwarding strategies. Each forwarding candidate ranks itself and its neighbors (who affirmatively or potentially received the message already) by distance to the centroid of neighbors in need of the message, to assign different priority in forwarding among neighboring nodes and remarkably suppress unnecessary retransmission, while enabling best nodes to transmit the packet without waiting. Kawamoto et al. [18] focused on mobile ad-hoc network based Cyber-physical Systems as a method to connect many kinds of wireless mobile devices for facilitating the next generation Cyber-physical Systems. The study uses each node to forward route discovery message that also includes its mobility and position of the adjacent nodes. After receiving the returned message, including the information of the node such as the position and the moving speed from the neighbors, a new topology is constructed according to the neighbors' information. After that, the optimal topology reconstruction interval and the redundant transmission range are calculated. Then, each node sets its transmission range, and keeps the transmission range during the time equal to maximum. The outcome of the study was evaluated using energy consumption, interval of topology construction, and energy expanded ratio. Skjelsvik et al. [19] have presented an event notification system for MANET essentially targeting the emergency condition. The study also introduces knowledge management to handle ontologies, resource management, security and privacy management, and watchdogs. R. Singh et al. [20] evaluated the performance of DSDV and DSR routing protocols for ad hoc networks Experimental results showed that DSR perform better for Packet Delivery Fraction as well as Throughput.

C. E Perkins et al. [21] proposed the on-demand routing protocols DSR and AODV, before sending a packet to the destination, discovers a route. Route maintenance is invoked when a node detects a link failure. C.A.F Cortes et al. [22] have worked on a middle system for performing enhancing routing behaviour with service delivery in heterogeneous MANET. P.Li et al. [23] study the ESCC (Energy- and Spectrumefficient Cooperative Communication) problem by exploiting the benefits of cooperative communication (CC) for mobile multimedia applications in multi-channel wireless networks. R. Chowdhuri et al. [24] modified Adhoc on demand Distance Vector to evaluate throughput, packet delivery ratio, and routing overhead. Hence, various techniques exists that has attempted in the past to enhance the routing performance in Mobile adhoc network. Zhenqiang Ye et al. [27] presents a frame work for Robust Secure, reliable routing in mobile adhoc networks. Javad Vazifehdan et al. [28] propose novel energy-aware routing algorithms .It considers the energy consumption and the remaining battery energy of nodes as well as the quality of links to find energy-efficient and reliable routes that increase the operational lifetime of the network. The proposed algorithm has higher routing overhead M Bheemalingam et al. [29] presents an Energy aware node disjoint multipath routing in Mobile Ad Hoc Networks

\subsection{Problem Discussion}

On the event of mobile node failures owing to the energy depletion in MANET, the research towards solving such node failure issues were found to be solved by routing protocols like Adhoc OnDemand Distance Vector routing protocol (AODV), Dynamic Source Routing (DSR), Destination-Sequenced

EASR: Graph-based Framework for Energy Efficient Smart Routing in MANET using... (Ramanna Havinal) 
Distance-Vector Routing (DSDV), and Zone Routing Protocol (ZRP). In proportion, it can be seen from previous studies that the majority of such techniques have used AODV and DSDV mainly. Hence, a research question lies-how much energy efficiency, they can give to MANET?. This question is quite difficult to answer as majority of the research has considered a specific environment or applications and not much generalized. With such routing protocols existing in literature archives, effectiveness and benchmarking outcomes are yet to be explored. Owing to the problems of dynamic topology, the mobile nodes in MANET are very difficult to be ascertained of its next position and hence the nodes are always overloaded with either data packets or control message from other neighboring nodes to establish communication links. Therefore, every node needs to spend extra energy for handling queries in traffic where the situation turns worst in large scale MANET. The prime challenge lies in preserving energy while active communication is carried out.

The primary benefits of AODV routing protocol are that it mechanizes the minimal routes that are congested inspite of using the shortest path that highly supports both multicast as well as unicast packet transmission even in uniform mobility. However, the prime disadvantage in AODV is that there is a higher likelihood of route validation expiry. The prime cause for this problem is mobility of nodes and their variable data transmission rate from one to other nodes consuming enough energy doing so. With the maximization of network size, performance metrics degrades, and so are energy factors using AODV routing protocol. Another limitation found in AODV is that it will need the condition that the mobile nodes in the transmission area should determine the broadcasting identity of other mobile nodes too.

One of the conflicting issues in DSDV is that it doesn't maintain load of traffic in large scale MANET and delay. Hence, it can be said that neither AODV nor DSDV should be considered as most efficient energy effective routing protocol till date, as it even doesn't address the basic QoS issues in MANET. Hence, there is a need of formulating a new routing protocol with completely new considerations and technical assumption that can ensure energy effectiveness in MANET. In this study, we have identified the issues pertaining to energy effectiveness in the MANET and proposed a new energy aware algorithm as it enhances the network lifetime of the MANET. The proposal of the idea exhibits routing model and graph theory to sort out the energy efficiency to reach the primary goal of mobile adhoc network with large scale environment

\subsection{Proposed Solution}

The proposed model presents a framework that could perform energy efficient routing in Mobile Adhoc Networks (MANET). In our previous study, a model was presented for the same cause using physical layer (PHY) optimization [25]; this work focuses much on the routing algorithm as it is believed that if the routing strategy is enhanced then energy dissipation among the mobile nodes could be controlled to a large extent in MANET. Usually, in MANET, the node acts as source point, destination point, as well as intermediate point to perform a data dissemination process. Because of undertaking of multiple operations, the buffer of the node is usually overloaded that results in congestion among the traffic for which reason a node depletes an extra amount of energy to forward the data packet effectively to destination point. Hence, the prime contributions of the proposed model are as follows:

1) A novel Energy-Aware Smart Routing (EASR) protocol is presented that uses geometrical approach and graph theory to forward the data packets in MANET.

2) Path Redundancy Metric is formulated for large traffic scenario to incorporate the multihop data forwarding process in MANET.

3) The outcome of the study is compared with AODV and DSDV protocol that are frequently adopted routing protocols in MANET for energy efficiency.

The prime goal of the study is to build an energy aware technique; however, better enhancement could be achieved if a novelty in the routing is established. Unfortunately, because of dynamic topology, such issues existing in present routing protocols couldn't be mitigated. Therefore, a novel routing technique is highly required that is completely new from the all the existing system. Hence, the study presents a geometrical based approach that mechanizes a new routing protocol considering a new type of node called as anchor nodes (AN). The prime objective of introducing anchor node is to assist in performing routing exclusively for heavy and congested traffic. The proposed EASR protocol considers anchor node to be fixed in one location and assists the mobile node to establish a route which in another case could have been not possible. Let us quote a pictorial illustration for understanding the behavior of our anchor nodes in MANET. Consider a possible scenario Figure 1(a), which show three nodes (Node-1, Node-2, and Node-3) with respective position $\left(x_{1}, y_{1}\right)\left(\mathrm{x}_{1}, \mathrm{y}_{1}\right),\left(\mathrm{x}_{2}, \mathrm{y}_{2}\right)$, and $\left(\mathrm{x}_{3}, \mathrm{y}_{3}\right)$. Also consider that each of the three nodes have their transmission range $R_{1}, R_{2}$, and $R_{3}$ (as in case of heterogeneous MANET) along with certain energy levels specific to each node. Let us consider a possible routing need of Node-1 as a source point to forward a data packet to Node-2 (as a destination point). In this case, it is impossible at time instant $t$ to establish routing between Node-1 and Node-2 as they are not in transmission range of each other or neither there is any 
intermediate mobile node to assist in data forwarding. Therefore, in this case, Node-1, in existing system has to perform two task viz. i) perform re-route discovery and find another alternative route or ii) use some delay-tolerant protocol [26]. However, in both cases there is a higher probability of energy dissipation that may also tend to reduce the lifetime of the network too. Moreover, owing to dynamic topology characteristics of MANET, there is a higher possibility that Node-2 moves further away from the existing position, which may even result in failure of delay tolerant protocols in MANET.

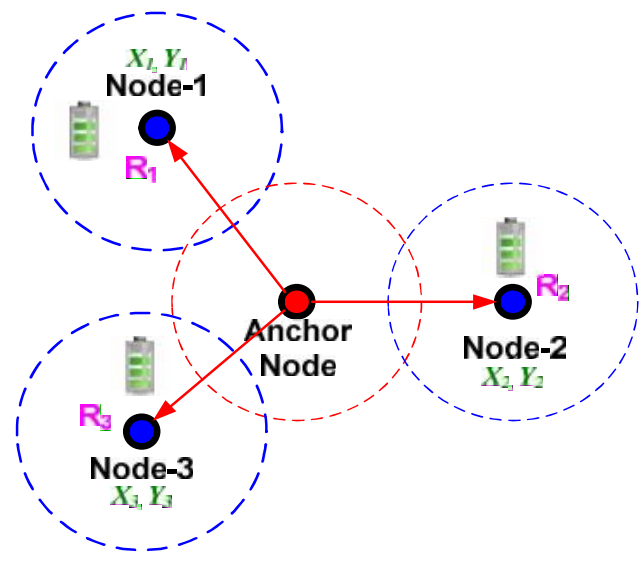

(a)

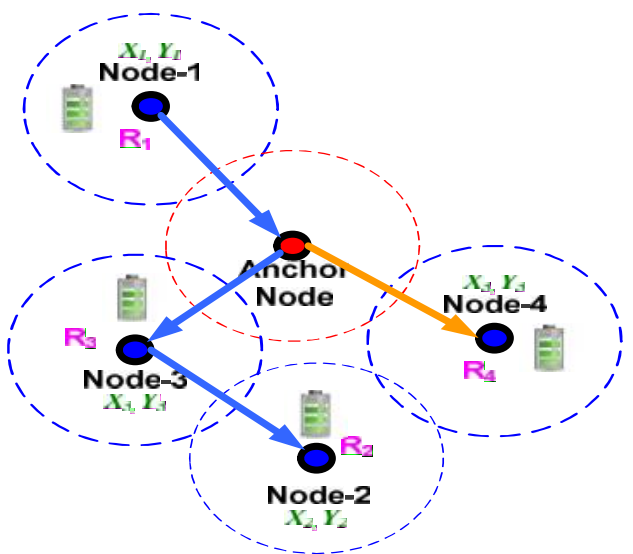

(b)

Figure 1. Illustation of Route Assistive behavior of Anchor Node

Therefore the proposed EASR protocol introduces AN to ensure that Node-1 should always be able to perform communication with Node- 2 . The AN uses a time slot to store all the mobile nodes' respective information pertaining to the position and residual energy. In Scenario (a), the AN stores these two information for Node-1, Node-2, and Node-3. Figure 1(b) shows a possible scenario, where Node-2 has changed its position and came in the proximity of Node-3 as well as Node-4. As Anchor node has previous time-stamped information about the position and residual energy information of Node-3 as well, hence, the routing will be possible from Node- 1 to Node- 2 in this situation. However, as it can also be seen that Node- 4 is also in proximity of Node- 2 while it is being recently registered by AN in time $t+1$ seconds, so it will be in the queue, for this reason, the communication overhead is reduced from Node- 4 . The effectiveness of this technique is that in case there is a possibility of an unstabilized link between Node-3 and Node-2, so the proposed system discards Node-3 and chooses Node-4 to perform routing. Therefore, it can be seen that at any position of the mobile nodes, a smart and cost effective routing is established that tremendously reduces re-transmission as well as re-route discovery process thereby preserving exponential amount of energy dissipation. Not only this, the proposed system also reduces the load of data dissemination from the mobile nodes and concentrates on establishing better and effective stabilized routes. The next section will illustrate further the formulation of EASR protocol to have better visualization of the methodologies.

\section{RESEARCH METHOD}

The proposed routing model is designed keeping the future applications in mind. The consideration of Anchor nodes(AN) is discussed in the previous section. In this section, we discuss that AN was located in specific zones termed as availability zone (AZ). The term "Availability Zone" is actually originated from the cloud conceptualization in Amazon Web Services [30], which means specific regions where the servers are positioned for performing better routing and extending better QoS factors to the ubiquitous users. Similarly, the study uses the term AZ to represent the static location of the AN, where number of AZs are categorized into primary AZs, secondary AZs, tertiary AZs etc. The proposed system considers two types of routing schema termed as i) Primary routing setup and ii) Secondary routing setup. The prime responsibility of both the routing schema is to ensure that data packet, definitely reaches its destination node at any cost with greater degree of reliability and packet integrity.

\subsection{Primary Routing Schema}

Figure 2 shows the primary routing schema. The rectangular box in the above figure represents th simulation area, where $n$-numbers of nodes are distributed in a random fashion and node movement is 
accomplished using random way point mobility model. Consider that red node represents a source node who is interested in forwarding the data packet to a specific destination node. For avoiding messy pictorial representation, we don't highlight the other mobile nodes in the simulation area. The source node forwards the query control message to one of the nearest primary availability zone as shown in Figure 2. After receiving the query, the primary AZs forward the query message to nearest primary AZs. If the task query for the path is met, the search terminates or else, the search is moved to secondary AZs. The same operation for searching the destination node is performed while in secondary AZs. It is to be noted that the search space for this discussion is limited to cardinality of $A Z=3$, however, it can be increased or decreased based on scale of applications in MANET. The search terminates if the destination node is reached, or else, the search continues for the secondary AZs. The design of the algorithm considers two types of routing cycle, e.g. i) the first routing cycle is estimated from the single route established between one source and one primary availability zone $\left(\mathrm{P}_{\mathrm{AZ}}\right)$ and then from that specific $\mathrm{P}_{\mathrm{AZ}}$, route is established among the entire component $\mathrm{P}_{\mathrm{AZ}}$ available. As AZs are basically the zone with availability of AN, so less energy dissipation is expected.

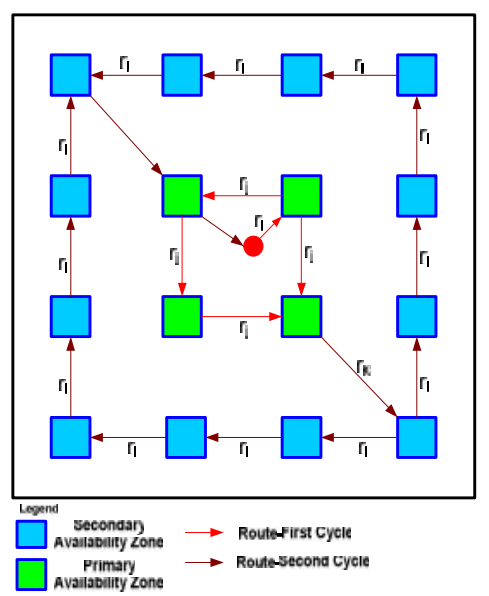

Figure 2. Primary Routing Set up

While the second cycle of routing is done by switching from $\mathrm{P}_{\mathrm{AZ}}$ to $\mathrm{S}_{\mathrm{AZ}}$ and the same routing process is performed. Similarly, switching from $\mathrm{S}_{\mathrm{AZ}}$ to $\mathrm{T}_{\mathrm{AZ}}$ can be performed depending on the load of the queries and accomplishment of the queries. The algorithm descriptions of the routing model are as follows.

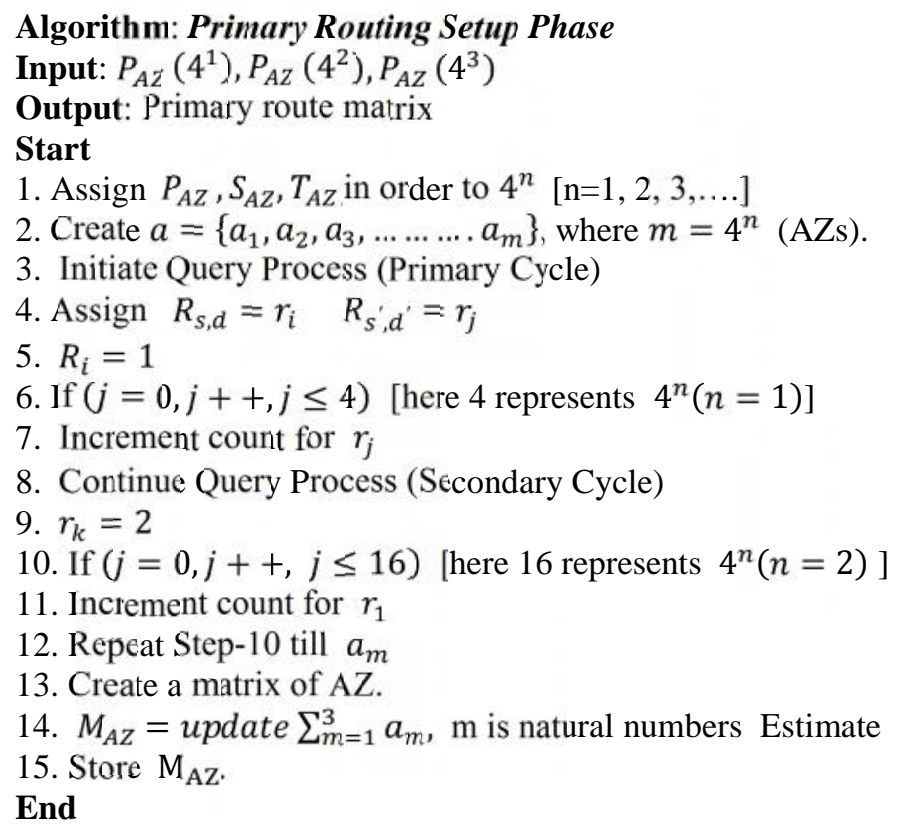

\subsection{Secondary Routing Schema}

The pictorial illustration of the routing scheme for secondary AZs is highlighted in Figure 3. 


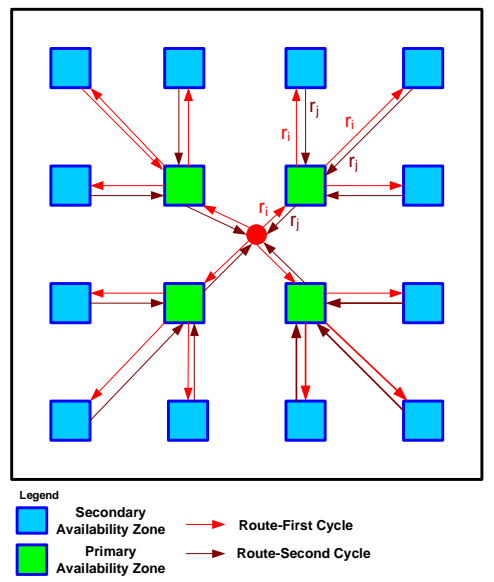

Figure 3. Secondary Routing Set up

In the secondary routing schema, we use the same primary and secondary AZs. This is a sequential phase, which will initiate only when primary routing setup is accomplished. The search space starts from the same position of source node which was in primary routing set up phase. However, the orientation of the routing totally changes in this phase. In secondary routing schema, the communication starts from primary AZs to secondary AZs. However, if it reaches the final secondary AZ, the retraced route path is set for the second cycle of routing. The secondary routing is an associated function of the proposed routing model, that makes the AN to perform a dual operation to ensure the existence of the destination node at any cost. The sample routing phenomenon is exhibited in Figure 3, where $r_{i}$ is the route vector connecting the AN from $\mathrm{P}_{\mathrm{AZ}}$ and $\mathrm{S}_{\mathrm{AZ}}$ and simultaneously extends to $\mathrm{T}_{\mathrm{AZ}}$. Whereas, $=r_{j}$ is the route vector that retraces its path back from $\mathrm{T}_{\mathrm{AZ}} \rightarrow \mathrm{S}_{\mathrm{AZ}} \rightarrow \mathrm{P}_{\mathrm{AZ}}$ provided the destination node exists, where the query message can be transmitted. In the given example, it can be seen that one $\mathrm{P}_{\mathrm{AZ}}$ could be possibly connected by $3 \mathrm{~S}_{\mathrm{AZ}}$ in one routing instance. Hence, the route from source $s$ to destination $d$ can be represented by $R_{s, d}$ may represent connectivity between any two AZs. Therefore, the algorithm represents final route establishment from one source to destination. The algorithm for secondary route setup phase is shown below:

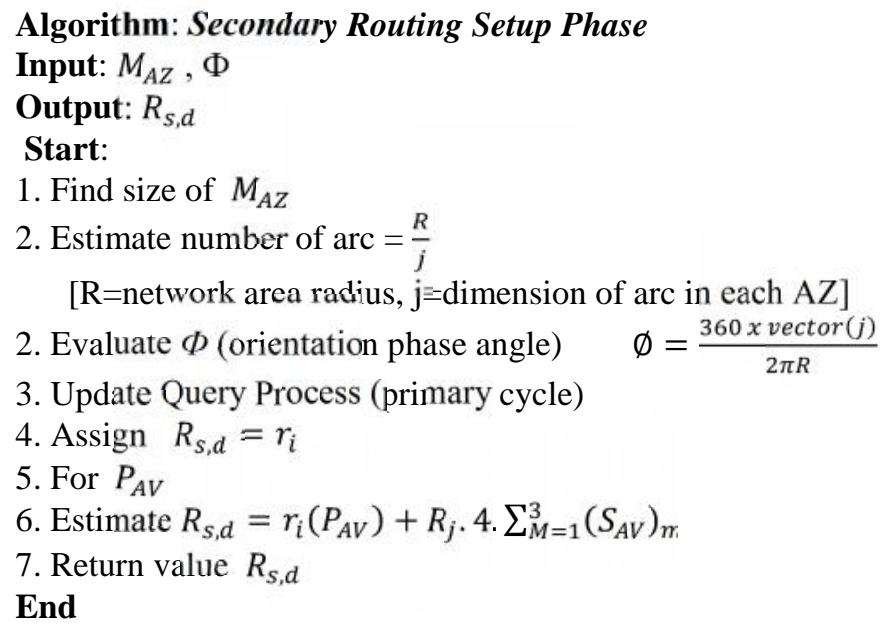

\subsection{Graph Theory Implementation}

In a MANET, data dissemination redundancy exploits the fact that messages are often delivered via multiple routes to compensate for packet loss, and that events are often observed by multiple mobile nodes. From the previous sections, it is understood that our model ensures $100 \%$ of data delivery provided,it is assumed that simulation is performed for a finite set of mobile nodes only. However, due to consideration of multiple availability zones, there is quite a possibility that redundant data are routed internally, although an algorithm reduces the overhead to $80 \%$. Therefore, we apply graph theory to overcome such issues of data redundancies. 
A network can be modeled as undirected graph. Therefore Consider $G=(V, E)$ is a directed graph where the vertices $\mathrm{V}$ represents mobile nodes and $\mathrm{E}$ represents edges or single hop communication vector in the considered scenario. The query information that is collected by the mobile node $S \in N$ (N=number of mobile nodes) is forwarded over multiple AZs $a_{1}, a_{2}, a_{3}, \ldots \ldots a_{m}$ to destination node $d$. For overcoming the problems of data packet loss (owing to energy depletion), the system will attempt to forward query information using a set of routes using multihop communication system, where each route can be depicted by the corresponding n-tuple of data forwarding mobile nodes. Another critical observation of the routing model is that due to the availability of multiple number of AZs (as well as ANs), it gives rise to multiple number of flows of the graph. Hence, we attempt to use advance graph theory to find the maximum graph flow. This section will illustrate a technique on the robust and scalable metric for redundant routes from AZs.

We formulate the condition that a mobile node exists in the simulation area that is associated with all the routes between sources and destination $d, G_{(s, d)}[V, E]$ becomes disconnected after switching the AVs. Therefore, we consider the unit value as length of graph's minimum vertex cut. We apply further condition that minimum vertex cut of a graph is equivalent to the maximum flow of the graph. This condition will also mean that the quantity of node-disjoint paths between the sources and destination $d$ in $G_{(s, d)}[V, E]$ is equivalent to 1 . The study will therefore use maximum flow algorithms to evaluate the quantity of nodedisjoint path more robustly and precisely. We then apply the theory of maximum flow in flow network [31] for evaluating the quantity of the edge-disjoint paths for $s$ and $d$ in the graph.

The Figure 4 exhibits the level two data flow diagram, where the sub-process of graph metric formulation is shown for decomposition. It will now derive an efficiently computable metric for redundant paths based on the notion of a query message transfer. If a node exists that is part of all paths, then graph becomes disconnected after removing this node. Thus, the size of the graph's minimum vertex cut is 1 . Applying theory of maximum flow in flow network will ensure the number of node-disjoint paths between source and destination is equal to 1. Ford Fulkerson Algorithm [32] is applied to find out the number of edgedisjoint paths for a source-destination pair in a weighted directed graph. Finally, edge weights are defined because maximum flow algorithms operate on weighted graphs.

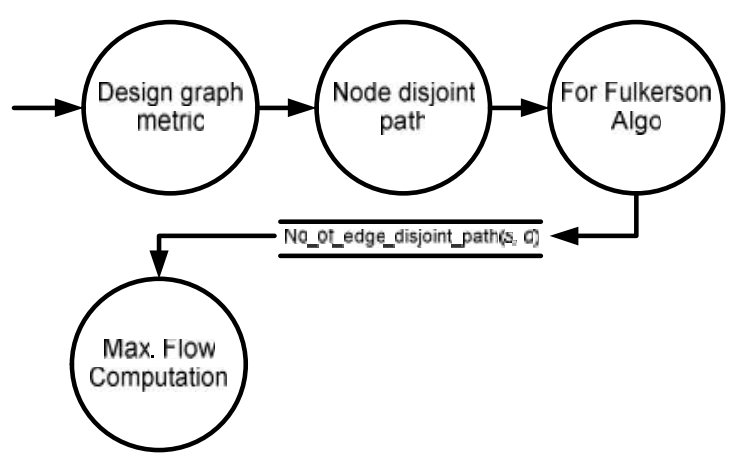

Figure 4. Evaluation of Max flow

A sparse matrix is created that represents a directed graph. Nonzero entries in the matrix G represent the capacities of the edges. The system also considers a parameter called as Capacity Value which is basically a column vector that specifies custom capacities for the edges in matrix G. It must have one entry for every nonzero value (edge) in matrix G. The order of the custom capacities in the vector must match the order of the nonzero values in matrix $G$ when it is traversed column-wise. The system will use a Ford Fulkerson algorithm. Time complexity is $\mathrm{O}\left(\mathrm{V}^{*} \mathrm{E}^{2}\right)$, where $V$ and $E$ are the number of nodes and edges respectively. Finally, implementation is carried out in calculating the maximum flow of directed graph $G$ from node $\mathrm{S}_{\text {node }}$ to node $\mathrm{T}_{\text {node }}$. Input $\mathrm{G}$ is a sparse matrix that represents a directed graph. Nonzero entries in the matrix $G$ represent the capacities of the edges. Max $_{\text {flow }}$ is the output of maximum flow and Flow Matrix is a sparse matrix with all the flow values for every edge. Flow Matrix $(p, q)$ is the flow from node $p$ to node $q$. Output flow cut is a logical row vector indicating the mobile nodes connected to $\mathrm{S}_{\text {node }}$ after calculating the minimum cut between $S_{\text {node }}$ and $T_{\text {node }}$. If several solutions to the minimum cut problem exist, then graph cut is a matrix. The steps of performing this are:

In this, the system initially creates a directed graph with a specific number of mobile nodes (vertices) and edges. The maximum flow of the graph is created. The graphs as well as the graph capacities 
are evaluated along with maximum flows. The next phase of the study was to design Ford Fulkerson (FF) Algorithm as below;

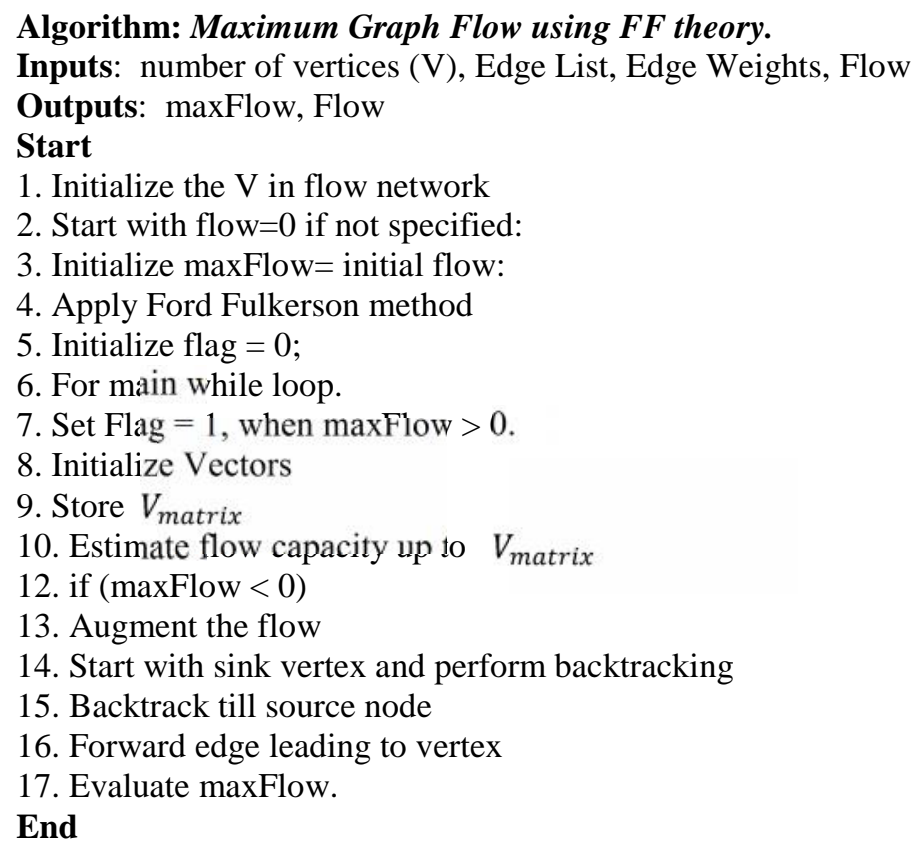

The above algorithm considers the input of 2 row matrix of directed edges where vertices are indexed from 1 as source to the destination node. The second important input argument will be weights of the routes that is represented by the column vector of corresponding weights. It is to be noted that weight metric is equivalent to capacity of edge in the graph logic applied in the proposed system. The third important input argument is an admissible flow to depict the communication channel in terms of established routes. The algorithm after applying the Ford Fulkerson method generates maximum flow (maxFlow) of the graph which is always a positive value depicting the maximum network flow of the graph. The final outcome of the algorithm generates a cumulative flow of an admissible flow of the network which according to the condition is always equivalent to the max-flow. Hence the algorithm successfully can generate the maximum flow in a flow network, where the flow network depicts the MANET system. Finally, the algorithm considering the network and graph based constraint for evaluating the cumulative maximum flow of the graph can be depicted as follows

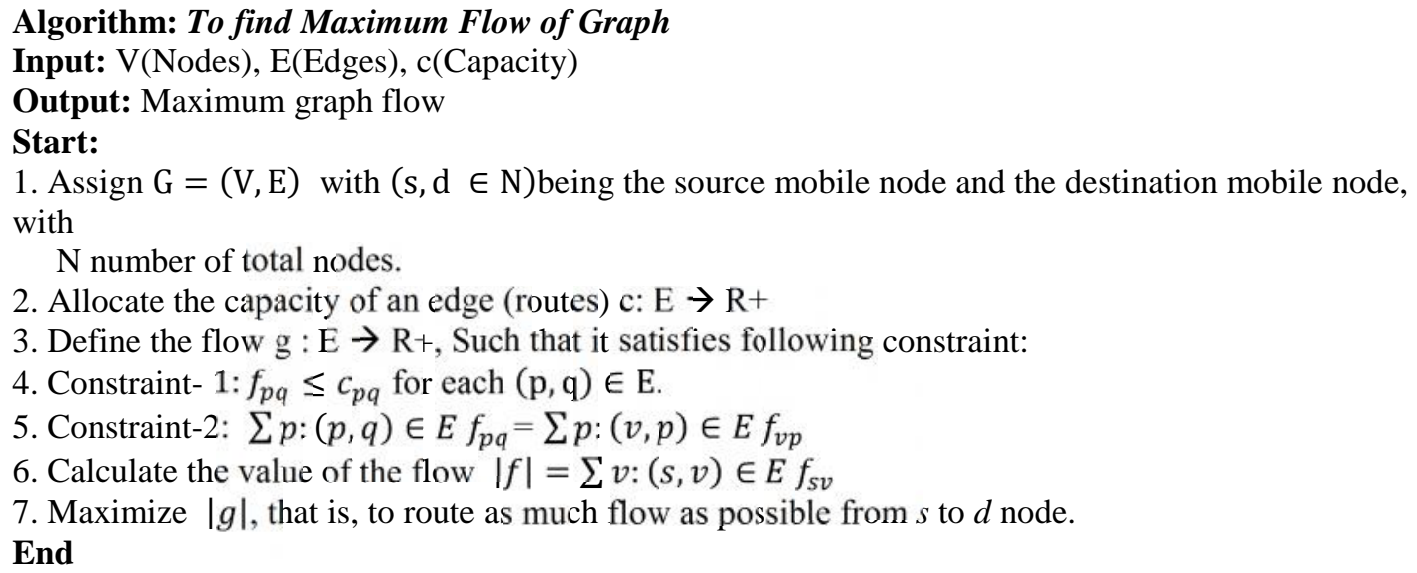

The above algorithm is responsible for finding the maximum flow of the graph. After the common assignment of the vertices (mobile nodes) along with routes (edges) using graph theory, the weights are allocated as it depicts the maximum quantity of the network flow that can pass through any routes (or edges). The algorithm is designed considering two constraints, e.g. the first constraint is related to the capacity of the network as the flow value of any edge cannot be higher than its capacity at any cost. The second constraint considered in the algorithm is the conservation of the network flow. The second constraint of the algorithm is 
evaluated with the sum of the network flows entering a mobile node (or vertices) that should be equivalent to the consolidated summation of the network flow exiting from a mobile node. However, this assumption doesn't consider the same for source and destination node to avoid anomaly of the query message. Finally, the algorithm computes the flow $g$ that essentially represents the quantity of the network flow passing from one source mobile node to another destination mobile node.

\section{RESULTS AND DISCUSSION}

The proposed routing model in MANET is implemented with 32 bit Windows OS with $1.84 \mathrm{GHz}$ Processor. The design environment is selected in Matlab. The mobile nodes use IEEE 802.11 radio and MAC model with a bit rate $11 \mathrm{Mbps}$ and each radio range is approximately a disc with a 250 meter radius. The size of each simulation universe is chosen to maintain an average node density of around 100 nodes per square kilometer. Each simulation runs for 300 simulated seconds. Each node moves using a random waypoint mobility model. The node chooses a random destination and moves toward it with a constant speed chosen uniformly between one and a maximum speed $(10 \mathrm{~m} / \mathrm{s}$ unless noted otherwise $)$. When the node reaches the destination, it chooses a new destination and begins moving toward it immediately. All simulations use a pause time of 0 second. For two location service protocols, we chose a beacon period of 2 seconds and the value update period was fixed at 25s. The metrics Dissipated Energy, Delay Estimation, and Completion Time analysis are performed for task allocation strategies designed in the proposed system. The energy efficiency of the proposed routing framework is evaluated by capturing the extent of energy being drained on each packet delivery process. The packet delivery ratio will include the estimation of the number of data packets being successfully transmitted from one source mobile node to the multiple mobile destination nodes in order to perform the data dissemination process in MANET. It is strongly believed that the concept of an availability zone introduced in this paper is quite novel idea and hence it becomes now a challenging task to perform a comparison of the proposed system with the existing research work. Hence, the outcomes cannot be directly compared with the existing system. But however, the proposed system uses graph theory, which was also implemented by numerous authors in past for enhancing the energy of the mobile nodes in MANET. From section 1.2, it was found that study discussed by authors in reference [23], [28], and [29] have used graph theory too for the same purpose. Hence, cumulatively, we consider all the other works inspired from graph theory as well as work done by authors in reference [23], [28], and [29] as existing system. The unique difference in proposed and existing system is presence and absence of concept of availability zone.

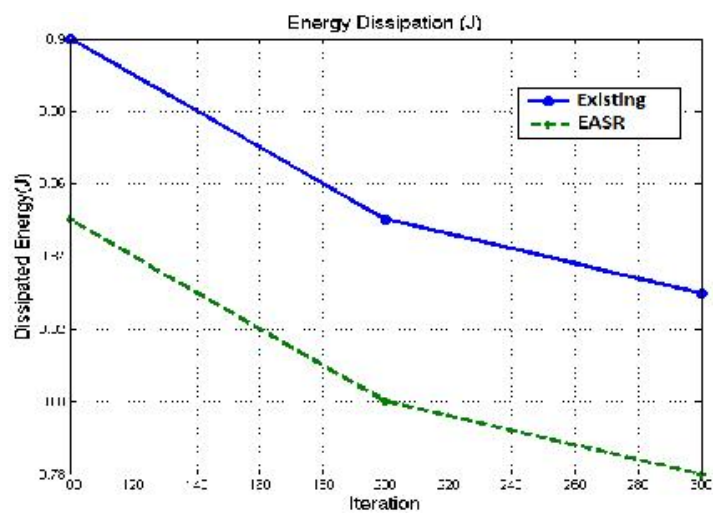

Figure 5. Outcome of Dissipated Energy

The plotting of the outcomes took place by recording the outcomes of dissipation energy, delay, and time consumed directly from the simulation results from both proposed as well as in the existing system. Figure 5 shows the amount of dissipated energy for the existing system and EASR and existing system. With the increasing rounds of iteration, the number of loads on each routes too increases. The existing system adopts the approaches of shortest path and performs energy intensive computation for routing over mobile nodes results in excessive energy dissipation. However, the current work considering AN accomplishes better energy efficiency as compared to the conventional routing technique that adopts the same system without AZ. By taking the responsibility of searching each route for routing the data packets to the destination node, AN conserves a significant amount of energy for the mobile nodes allowing more capability to disseminate 
the data. Incorporations of AZ not only assists in enhancing the routing decision but also significantly assists in minimizing the load on each mobile node to store and process the information resulting in lesser consumptions of energy.

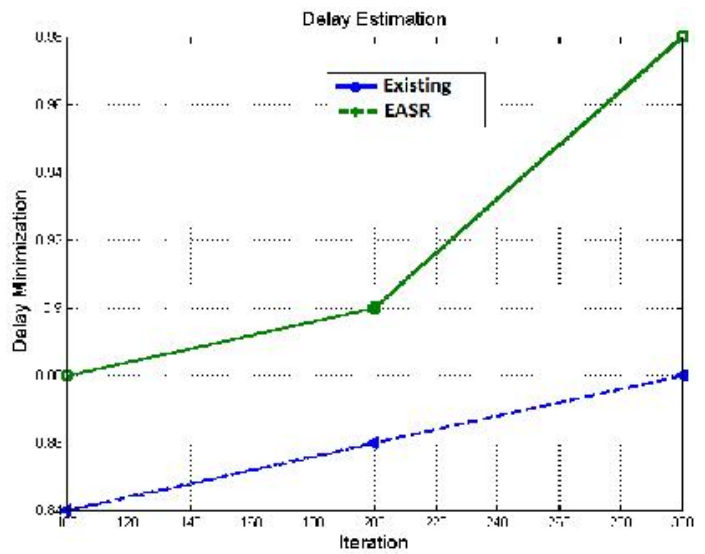

Figure 6. Outcome of Delay Estimation

The outcome of the study considers delay evaluation as another attribute that has been considered to evaluate the performance of the designed system. It estimates the total time difference taken to perform the data dissemination process in common MANET system (usually small scale network with all mobile nodes) of conventional data dissemination techniques and proposed technique. Figure 6 shows that there has been an extensive delay minimization accomplishment in the proposed system that outperforms the technique considering absence of AZ. However, due to constant need of update of task allocation policy, the minimization process is somewhat slower till 200 seconds of time instant of simulation, but it rapidly accumulates the speed after 200 second. In existing system the data holding time is not only longer but sometimes it may also lead to drop of data packet when the node reaches its culminative battery life. Hence, inclusion of AZ significantly increases the data dissemination process leading to minimization of data holding time by any mobile node.

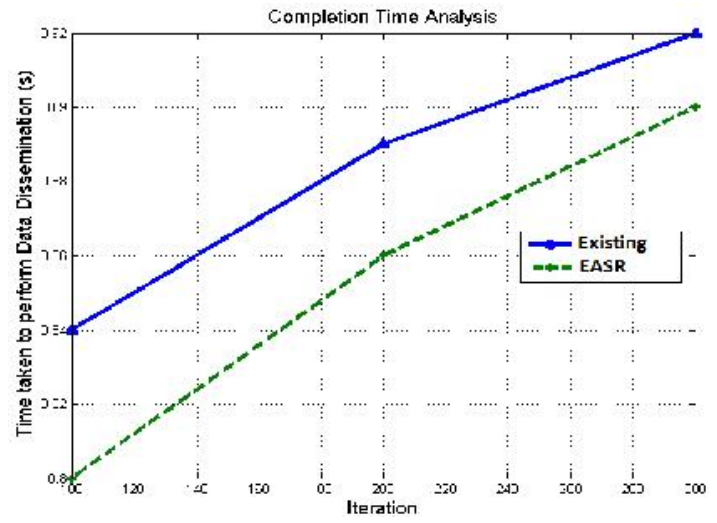

Figure 7. Outcome of Task Completion Time

The final parameter for checking the performance evaluation is task completion time. Figure 7 highlights that the time taken to perform the data dissemination and route establishment process is quite faster in presence of AZs as compared to conventional existing technique without AZs. The task query pattern is selected for visualizing and studying the efficiency of the query and update mechanism of two protocols. Every mobile node in the network initiates a certain number of task queries using AZ to look up the position information of randomly destinations at times randomly distributed between certain intervals of time. Also, if a query is not successful, no retransmission is initiated. For estimating the preciseness of the 
query reply, when the query reply is received, each source mobile node sends a single data packet of size in bytes to that destination mobile node using the AZ; this is done after finding a optimal route to destination end. Hence the performance is highly optimized for the proposed system. Hence, in order to have more clarity of impact of AZ on the energy efficiency, Figure 8 shows, although inclusion of AZ increases energy efficiency, but that will not mean higher dependencies on AZ. The outcome shows that availability of just 2 AZ can offer better energy conservation in MANET.

Therefore, Figure 8 shows that when the AZs are used, the rate of energy conservation (energy $(\mathrm{J})$ per seconds of data dissemination) is highly optimized leading to longer network lifetime. Hence, the proposed protocol can successfully be implemented in the advanced version of applications in MANET, where numbers of mobile nodes are higher. The proposed system can comfortably tackle the communication issues of large scale MANET with better energy efficiency. However, when the availability zone is removed, the performance starts degrading with increasing iteration where the loads are increased for every cycle of the data dissemination process. The next section will discuss about the performance comparative analysis of the proposed routing model.

It has been noticed in the review of literature that the majority of the prior studies has used AODV as well as DSDV as the prime contributors of energy efficiency attributes towards designing a better routing protocol in MANET. However, routing scheme with an aid of AODV or DSDV was never experimented on any past experiments by considering any additional parameter in MANET that is responsible to store and process significant information e.g battery, location, energy etc. Hence, may be the existing solution may sound better energy efficiency on dense network, but it will never be applicable on sparse network where the mobile of the mobile nodes is highly invariable factor (variable speed). As majority of the work done in the past (Section 1.2) have adopted uniform speed, and typical energy-conservation technique therefore the existing system doesn't ensure enough energy efficiency for sparse network of large dimensionality and on variable node mobility scenario.

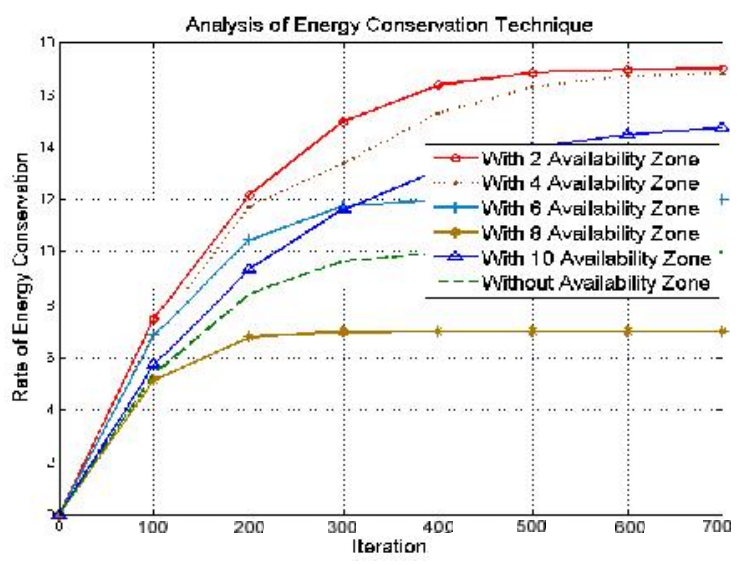

Figure 8. Outcome of Energy Conservation Technique

The proposed system furnishes novelty by introducing the new actor called as AZ or availability zone, where ANs are located. One of the interesting property of this algorithm is that it ensures a better and reliable data dissemination process and borne the majority of the load of routing activity on themselves (AZ). Hence, it is positively expected that the proposed model drastically restores energy of the mobile nodes. Till date, none of the existing research study has ever considered an external actor like what we did in our work called as Availability Zones. This novel concept has assisted to reduce the energy consumption among the mobile nodes to greater extent as Anchor Nodes performs all the task of routing that automatically updates along with mobility pattern of the MANET. Probably, the proposed system can be said to be successfully coping with dynamic topology of MANET. 


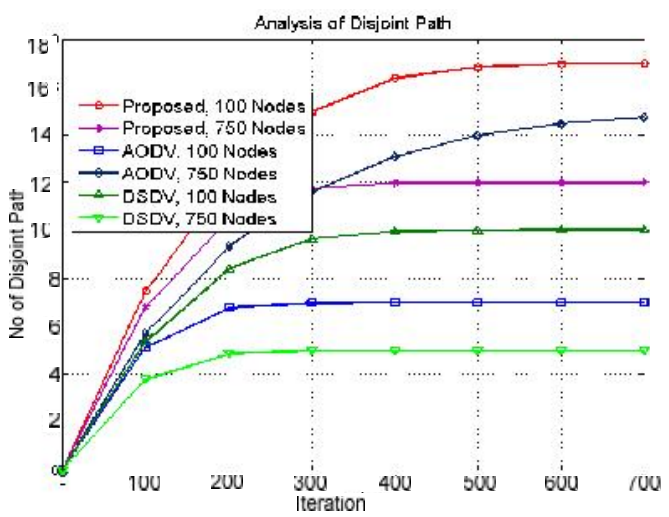

Figure 9. Analysis of Node disjoint path

Figure 9 shows the analysis of the disjoint sets considering the proposed data dissemination process in MANET, along with conventional AODV and DSDV routing protocols. It can be seen that with the incorporation of lightweight energy efficient algorithm using maximum graph flow, the proposed system performs better and has higher capability of evaluating number of disjoint paths. Considering the simulation with 100-750 nodes in 8 rounds of iteration, proposed system excels well in performance.

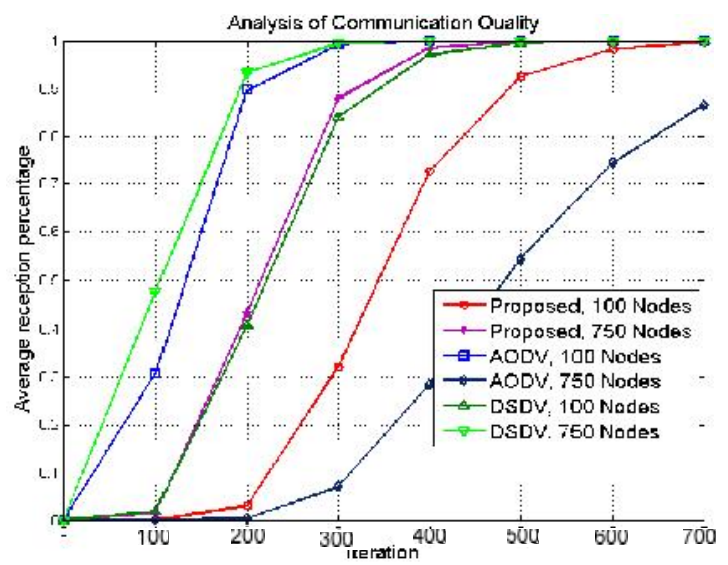

Figure 10. Analysis of Communication Quality

Figure 10 exhibits the analysis of the communication quality, where it can be seen that the proposed system performs almost equivalent to DSDV protocols considering 750 nodes and 100 nodes respectively. This will also mean that adoption of proposed system will be higher and it is more flexible in routing in public network in MANET. AODV perform better after proposed system. One interesting things is performance of proposed system increases with increase of iteration, which makes it best compared to DSDV and AODV routing protocols.

\section{CONCLUSION}

The proposed study introduces a novel topological based approach that uses completely novel mechanism of energy aware routing in mobile adhoc network. The study uses the concept of availability zone that is a group of anchor nodes passing the positional information about the adjacent mobile nodes in a particular time instant. Any sender node looking for performing routing will forward its query message to any one of the availability zone, which checks for the availability of the destination nodes by performing internal routing with other availability zones. The prime motive was to ensure energy conservation and efficient routing. The routing framework is formulated using the graph theory where graph maximum flow is conceptualized. The proposed system is also compatible for large scale MANET as the outcome shows better comparison with respect to frequently used AODV and DSDV. Although the literatures have shown better 
utilization of AODV, but the routing structure of the AODV is still having higher possibility of traffic overhead in large scale MANET and moreover none of the previous work has used any external factor to increase the connectivity and routing of the proposed MANET system. However, the proposed system can yield better results considering finite sets of mobile nodes and assumptions being done towards the functional design of AZ. The future work will emphasis on further enhancement of the same energy aware routing in MANET with further optimization scope.

\section{REFERENCES}

[1] J. Loo, J. Lloret Mauri, Jesús Hamilton Ortiz, "Mobile Ad Hoc Networks: Current Status and Future Trend's", CRC Press, Computers, pp. 1-538, 2012.

[2] D. Coudert, D. Simplot-Ryl, and I. Stojmenovic, “Ad-hoc, Mobile and Wireless Networks", Springer, Computers. pp.1- 498, 2008.

[3] Y. Zhang, L. T. Yang, J. Ma, "Unlicensed Mobile Access Technology: Protocols, Architectures, Security, Standards and Applications", CRC Press, Technology \& Engineering, pp. 1-424, 2008.

[4] Shivshankar, H. N. Suresh, G. Varaprasad, and G. Jayanthi, "Designing Energy Routing Protocol with Power Consumption Optimization in MANET”, IEEE Transactions on Emerging Topics in Computing, Vol. 2, No. 2, pp. 192-197, 2014.

[5] C. Aydogdu and E.Karasan, "An Analysis of IEEE 802.11 DCF and Its Application to Power-Efficient Relaying in Multi-Hop Wireless Networks", IEEE Transaction on mobile computing, Vol. 10, No. 10, pp. 1361-1373, 2010.

[6] A. E. A. A. Abdulla, Z. Md. Fadlullah, H. Nishiyama, and N. Kato, "On the Optimal Transmission Distance for Power-aware Routing in Ad hoc Networks", IEEE-International Conference on Computing, Networking and Communications, Wireless Ad Hoc and Sensor Networks Symposium, San Diego, USA, 2013.

[7] S. H. Lee, Y. Choi, S. Park, and S. H. Rhee, "An Power Efficient Power Control Mechanism for Base Stations in Mobile Communication Systems”, IEEE National Research Foundation of Korea (NRF), pp. 378-383, 2011.

[8] J. Zuo, C. Dong, H. V. Nguyen, S. X. Ng, L-L Yang, and L. Hanzo, "Cross-Layer Aided Energy-Efficient Opportunistic Routing in Ad Hoc Networks", IEEE Transactions On Communications, 2013.

[9] A. H. Ali, F. Kanwal, K. Bashir, "Centrally Coordinated Power Aware Route Selection for MANETs", IEEEInternational Conference on Open Source Systems and Technologies, pp. 87-90, 2013.

[10] T. Mukherjee, G. Varsamopoulos, and S.K.S Gupta, "Self-managing energy-efficient multicast support in MANETs under end-to-end reliability constraints", ELSVIER Computer Networks, Vol. 53, No. 10, pp. 1603-1627, 2009.

[11] S. Mangai, Velmurugan, and A. Tamilarasi, "A new approach to geographic routing for location aided cluster based MANETs", EURASIP Journal on Wireless Communications and Networking, Vol. 1, pp. 1-10, 2011.

[12] F. Correia, and T. Vazão, "Simple ant routing algorithm strategies for a (Multipurpose) MANET model", Ad Hoc Networks, Vol. 8, No. 8, pp. 810-823, 2010.

[13] Q. Fu, B. Krishnamachari, and L. Zhang, "DAWN: A density adaptive routing for deadline-based data collection in vehicular delay tolerant networks", IEEE Tsinghua Science and Technology, Vol. 18, No. 3, pp. 230-241, 2013.

[14] F. Li, X. He, S. Chen, L. Jiang, and Y. Wang, "Traffic Distribution of Circular Sailing Routing in Dense Multihop Wireless Networks", IEEE- Tsinghua Science And Technology, Vol. 18, No. 3, 2013.

[15] G. Jianli, L. Hongwei, D. Jian, and Y. Xiaozong, "HEAD: A hybrid mechanism to enforce node cooperation in mobile ad hoc networks", IEEE-Tsinghua Science \& Technology, Vol. 12, pp. 202-207, 2012.

[16] B. Roy, S. Banik, P. Dey, S. Sanyal, and N. Chaki. "Ant colony based routing for mobile ad-hoc networks towards improved quality of services", Journal of Emerging Trends in Computing and Information Science, Vol. 3, No. 1, pp. 10-14, 2012.

[17] J. Liu, Z. Yang, and I. Stojmenovic, "Receiver Consensus: On-time Warning Delivery for Vehicular Ad-hoc Networks", In Distributed Computing Systems (ICDCS), IEEE 32nd International Conferenc, pp. 386-395, 2012.

[18] Y. Kawamoto, H. Nishiyama, and N. Kato, "MA-LTRT: A Novel Method to Improve Network Connectivity and Power Consumption in Mobile Ad hoc based Cyber-Physical Systems", IEEE Emerging Topics in Computing, Vol. 1, No. 2, pp. 266-374, 2013.

[19] T. Plagemann, K. S. Skjelsvik, and V. Goebel, "Distributed event notification for mobile ad hoc networks", IEEE Distributed Systems computer Society Online, Vol. 5, No. 8, 2004.

[20] R. Singh, Dharmendra K Singh, and L. Kumar, "Performance Evaluation of DSR and DSDV Routing Protocols for Wireless Ad Hoc Networks", International. Advanced Networking and Applications, Vol. 2, No. 4, pp. 732-737, 2011.

[21] C. E. Perkins and E. M. Royer, “Ad-Hoc On-Demand Distance Vector Routing”, Second 1EEE Workshop on Mobile Computing Systems and Applications, pp. 90-100, 1999.

[22] C. A. F Cortés, G.S. Blair, and P. Grace, "Middleware An Adaptive Middleware to Overcome Service Discovery Heterogeneity in Mobile Ad Hoc Environments", IEEE Distributed System Computer Society, online. Vol. 8, No. 7, 2007.

[23] P. Li, S. Guo, J. Hu, "Energy-Efficient Cooperative Communications for Multimedia Applications in Multi-Channel Wireless Networks", IEEE Transactions on Computers, 2013.

[24] R. Chowdhuri, D. Barma M. K. Sen, "Comparison of Performance Metrics of DVR Protocol Using Node Remaining Energy and Aggregate Interface Queue Length”, Elsevier, 2013. 
[25] Ramanna. Havinal, G. V. Attimarad, M. N. Giriprasad, "Empirical Power Control Schema Using Physical Layer Optimization in MANET", IEEE Computer Society, ACM Digital Library, pp. 130-135, 2013.

[26] R. Lu, X. Lin, H.Zhu, "Pi: A Practical Incentive Protocol for Delay Tolerant Networks", IEEE Transactions On Wireless Communications. Vol. 9, No. 4, 2010.

[27] Zhenqiang Ye, Srikanth V Krishnamurthy, and Satish K Tripathi, "A frame work for reliable routing in mobile adhoc networks", IEEE Infocom 2003.

[28] Javad Vazifehdan, R. Venkatesha Prasad, and Ignas Niemegeers, "Energy-Efficient Reliable Routing Considering Residual Energy in Wireless Ad Hoc Networks", IEEE Transactions on Mobile Computing, Vol. 13, No. 2, 2014.

[29] M Bheemalingaihm, M. M Naidu, D Srinivasrao, G. Varaprasad, "Energy Aware node disjoing multipath routing in Mobile Ad Hoc Networks", Journal of Theoratical and applied information Technology, Vol. 45, No. 12, pp. 416$431,2009$.

[30] http://docs.aws.amazon.com/AWSEC2/latest/UserGuide/using-regions-availability-zones.html

[31] http://en.wikipedia.org/wiki/Max-flow_min-cut_theorem

[32] http://en.wikipedia.org/wiki/Ford\%E2\%80\%93Fulkerson_algorithm

\section{BIOGRAPHIES OF AUTHORS}

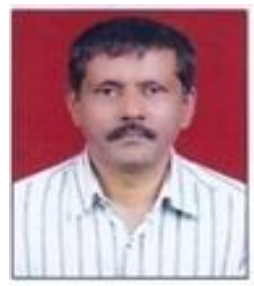

Ramanna Havinal received his B.E, M.E degree from Karnataka University, Dharwad, India in 1991, 2000 respectively. Currently he is pursuing his Ph.D from J N T University Anantapur, India. Presently he is working as Associate Professor in the Department of Electronics and Communication Engineering at College of Engineering Ambajogai, India. He is having more than 24 years of teaching experience His research areas are Wireless Communications, Wireless Networks, Digital Signal and Image processing. He has presented more than 15 papers in national and international conferences/Journals.

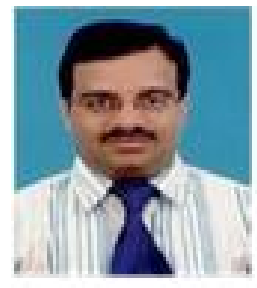

Girish V Attimarad received his B.E degree in 1993 from Gulbarga University, India, M.E degree in 1995 from Karnataka University, Dharwad, India and Ph.D degree in 2003 from Delhi University Delhi, India. Presently he is working as Professor and Head of the department of Electronics and Communication Engineering Department at Dayanand Sagar College of Engineering, Bengaluru India. He is having more than 22 years of teaching experience His research areas are Wireless Communications, Wireless Networks, Microwaves and Radar, Open wave guides. He has published many papers in national and international journals/conferences. He is a life member of ISTE,IE, IETE

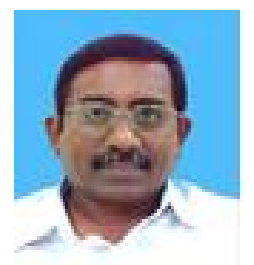

M N Giriprasad received his B.Tech degree in 1982 from JNTU College of Engg, Anantapur, India .M. Tech degree in 1994 from Sri Venkateswara University, Tirupati, India and Ph.D degree in 2003 from J N T University, Hyderabad, India. Presently he is working as Professor and Head of the department of Electronics and Communication Engineering at JNTUA College of Engineering, Anantapuramu, India. He is having more than 28 years of teaching experience. His research areas are Wireless Communications, Wireless Networks, Signal Processing, and Image processing, Biomedical Instrumentation. He has published more than 26 papers in national and international conferences. Around 55 papers published in national and international journals. $\mathrm{He}$ is a life member of ISTE, IEI and IEFN. 